\title{
Combination of Amniotic Membrane Stem Cell Metabolite Product (AMSC-MP) and Vitamin E for Photoaging
}

\author{
Retha, Sawitri, Rahmadewi, Afif Nurul Hidayati, Muhammad Yulianto Listiawan, Evy \\ Ervianti, Linda Astari, Cita Rosita Sigit Prakoeswa \\ Departement of Dermatology and Venereology \\ Faculty of Medicine Universitas Airlangga/Dr. Soetomo General Academic Teaching Hospital \\ Surabaya
}

\begin{abstract}
Background: It is hypothesized that combination of amniotic membrane stem cell metabolite product (AMSC-MP) and vitamin $\mathrm{E}$ after fractional $\mathrm{CO}_{2}$ laser as laser assisted drug delivery (LADD) provides better long-term effects on clinical improvement of photoaging. This is promising as an option for photoaging therapy in the future. Purpose: The study aimed to evaluate long-term effects of improving the degree of wrinkles, polarized black spots, ultraviolet (UV) black spots, pores, and skin tones in subjects who have received a topical mixture of AMSC-MP with vitamin $\mathrm{E}$ and $\mathrm{CO}_{2}$ fractional laser as LADD. Methods: Thirty adult women with photoaging who had been treated with topical AMSC-MP mixed therapy with vitamin $\mathrm{E}$ and $\mathrm{CO}_{2}$ fractional laser three times, were then given maintenance therapy, tretinoin cream $0.025 \%$ and sunscreen SPF 30. Observation of improvement of wrinkles, polarized black spots, UV black spots, pores, and skin tones were performed computer-stimulated photographs, Janus-II Facial Skin Scope System, three times every month for three months after the last treatment. Results: The evaluation of wrinkles, polarized black spots, UV dark spots, pores and skin tones from the assessment for three months after being receiving treatment of a topical mixture of AMSC-MP with vitamin $\mathrm{E}$ and $\mathrm{CO}_{2}$ fractional laser as LADD on photoaging obtained $p>0.05$. Conclusion: There was a long-term effect of the topical mixture of AMSC-MP with vitamin $\mathrm{C}$ and $\mathrm{CO}_{2}$ fractional laser on photoaging, and there was no side effect observed.
\end{abstract}

Key words: amniotic membrane stem cell metabolite product, vitamin $\mathrm{E}$, fractional $\mathrm{CO}_{2}$ laser, photoaging, long-term effect.

Correspondence address: Sawitri, Department of Dermatology and Venereology, Faculty of Medicine, Universitas Airlangga, Dr. Soetomo General Academic Teaching Hospital, Jl. Prof. Dr. Moestopo No. 6-8 Surabaya 60131, Indonesia. Phone: + 6231-5501609, e-mail: sawitri.rh@gmail.com.

\section{INTRODUCTION}

Skin aging is a complicated process resulting in several functional and aesthetic changes. There are two processes that induce skin aging, namely intrinsic and extrinsic processes. The extrinsic process is often called photoaging. Intrinsic factors are unavoidable and as a natural consequence of genetics over time. Photoaging causes premature skin aging caused by environmental factors such as sun exposure, air pollution, smoking habits, and poor nutrition. ${ }^{1,2}$ These elderly ages data can be related to the prevalence of photoaging, according to the study of Malvy and colleagues who examined 12,735 patients aged $45-60$ years in France, the highest prevalence of photoaging was the 55-60 age group compared to other age groups. $^{3}$

Vitamin E has antioxidant properties, and it is a widely used skincare industry. Vitamin $\mathrm{E}$ has been used for more than 50 years in experimental and clinical use in the field of dermatology. Most experimental studies have shown photoprotective, antitumorigenic effects, and the ability to stabilize the skin barrier. ${ }^{4}$ Vitamin $\mathrm{E}$ can significantly reduce erythema, edema, skin hypersensitivity related to UVB radiation, and UV-induced skin cancer. Topical vitamin $\mathrm{E}$ has emollient and non-irritating properties, permeation ability, and it has molecules that compatible with the skin. ${ }^{5}$

Therapeutic stem cell metabolites products is currently under development. Metabolite products are produced when stem cells are cultured and does not contain cell. Amniotic membrane stem cell metabolite products (AMSC-MP) contain several growth factors such as Epidermal Growth Factor (EGF), Transforming Growth Factor- $\beta 1$ (TGF- $\beta 1$ ), basic Fibroblast Growth Factor (bFGF), Vascular Endothelial Growth Factor (VEGF), etc. PM-AMSC also contains cytokines named Interleukin-4 (IL-4), Interleukin-6 (IL-6), Interleukin-8 (IL-8), and interferon (Effendy, 2017). Prakoeswa has examined 
the effectiveness of AMSC-MP in leprosy ulcer healing. The research obtained good results in the AMSC-MP mixture group with vitamin $\mathrm{E}^{6}$

Ablative fractional laser can facilitate the administration, and it is referred to as laser-assisted drug delivery (LADD), which use has been studied in the last decade for clinical practice. LADD damages stratum corneum, epidermis, and dermis for improved drug delivery. ${ }^{7}$ In general, LADD requires low energy to damage the stratum corneum in order to improve dermal penetration. ${ }^{8}$ The ideal parameters for creating a completely varied channel depend on the laser type, device model, and settings. Energy use for effective channel depth is currently under debate. ${ }^{9}$ Channel is expected to reach the superficial dermis layer for LADD in photoaging cases. ${ }^{10}$

PM-AMSC and vitamin E treatment after fractional laser as LADD is expected to improve photoaged skin and provide extended effect by facilitating drug penetration. PM-AMSC contains several growth factors and cytokines which improve skin rejuvenation. Vitamin E, as an antioxidant, also plays a role in skin rejuvenation. This study aimed to evaluate the long-term effects of topical mixture of AMSC-MP with vitamin $\mathrm{E}$ and $\mathrm{CO}_{2}$ fractional laser as LADD on photoaging.

\section{METHODS}

This study hypothesized that a combination of topical AMSC-MP and vitamin E treatment for three months could improve wrinkles, polarized spots, UV spots, skin tone, and pores due to photoaging in longterm duration of time.

This was a cohort study. There were 30 patients with Glogau 2 and 3 photoaged skins that had been treated for three months. The skins were examined monthly at the Cosmetic Division, Dermatology and Venereology Outpatient Clinic, Dr. Soetomo Hospital.

The participants were also provided with $30 \mathrm{SPF}$ sunscreen and tretinoin $0.025 \%$ cream as maintenance therapy during the treatment. Wrinkles, pores, polarized black spots, UV black spots, and skin tone were evaluated using Janus-II Facial Skin Scope (Janus). The evaluations were performed monthly, the first month (Janus 3), second month (Janus 4), and third month (Janus 5). The data were then processed using the SPSS version 17 program. This study has been approved by the ethics committee of Dr. Soetomo General Hospital Surabaya.

\section{RESULTS}

This was a 3-month observational cohort study, starting from June to August 2019 at the Cosmetic Division, Dermatology and Venereology Outpatient
Clinic, Dr. Soetomo Hospital. A total of 30 participants met the inclusion criteria, have signed informed consent and medical action, and did not have any exclusion criteria. The participants received the treatment monthly from January to April 2019.

All participants were female, and they were grouped according to age range. The age group was $46-55$ years; 11 people $(36,7 \%)$, followed by the $36-$ 45 years age group; 10 people $(33,3 \%)$ and the 56-65 years age group; 9 people $(30 \%)$. The youngest patient was 38 years old, and the oldest was 62 years old.

Glogau type 3 was observed in 16 participants $(53.3 \%)$ and type 2 was observed in 14 participants (46.7\%). Fitzpatrick skin type 5 was observed in 16 participants $(53.3 \%)$, and type 4 was observed in 14 people $(46.7 \%)$. There were 24 participants $(80 \%)$ resided in Surabaya, and the remaining 6 participants $(20 \%)$ resided outside Surabaya. All participants $(100 \%)$ had indoor activities every day. All participants $(100 \%)$ claimed that they routinely used sunscreen, while 4 participants $(13.33 \%)$ used umbrellas as additional protection. All subjects $(100 \%)$ also claimed that, in general, they exposed to the sun during their way to work. A total of 10 participants $(33.33 \%)$ often sunbathe in the morning. All patients $(100 \%)$ routinely used $0.025 \%$ tretinoin cream as maintenance therapy.

Table 2 shows the 3-month results of wrinkle evaluation using Janus of the monthly AMSC-MP and vitamin $\mathrm{E}$ treatment. There was no significant difference in the values of wrinkles between the third, fourth, and fifth Janus evaluation. This indicated the treatment effect on wrinkles was present.

Table 3 shows the 3-month results of polarized black spots evaluation using Janus of the monthly AMSC-MP and vitamin E treatment. There was no significant difference in the values of polarized black spots between the third, fourth, and fifth Janus. This indicated the treatment effect on polarized black spots was present.

Table 4 shows the 3-month results of UV blackspots evaluation using Janus of the monthly AMSCMP and vitamin E treatment. There was no significant difference in the values of UV black spots between the third, fourth, and fifth Janus. This indicated the treatment effect on UV black spots was present.

Table 5 shows the 3-month results of skin tone evaluation using Janus of the monthly AMSC-MP and vitamin $\mathrm{E}$ treatment. There was no significant difference in the skin tone values between the third, fourth, and fifth Janus. This indicated the treatment effect on skin tone was present.

Table 6 shows the 3-month results of pore 
evaluation using Janus of the monthly AMSC-MP and vitamin $\mathrm{E}$ treatment. There was no significant difference in the pores between the third, fourth, and fifth Janus. This indicated the treatment effect on pores was present. No side or adverse effect observed after the treatments (Table 7).

Table 1. Demographic data of research subjects

\begin{tabular}{|c|c|}
\hline Variable & $\mathrm{n}(\%)$ \\
\hline \multicolumn{2}{|l|}{ Sex } \\
\hline Male & $0(0)$ \\
\hline Female & $30(100)$ \\
\hline \multicolumn{2}{|l|}{ Age } \\
\hline $36-45$ years old & $10(33.3)$ \\
\hline $46-55$ years old & $11(36.7)$ \\
\hline $56-65$ years old & $9(30)$ \\
\hline \multicolumn{2}{|l|}{ Glogau } \\
\hline Type 2 & $14(46.7)$ \\
\hline Type 3 & $16(53.3)$ \\
\hline \multicolumn{2}{|l|}{ Fitzpatrick skin } \\
\hline Type 4 & $14(46.7)$ \\
\hline Type 5 & $16(53.3)$ \\
\hline \multicolumn{2}{|l|}{ Domicile } \\
\hline Surabaya & $24(80)$ \\
\hline Outside Surabaya & $6(20)$ \\
\hline \multicolumn{2}{|l|}{ Occupation } \\
\hline Indoor & $30(100)$ \\
\hline Outdoor & $0(0)$ \\
\hline \multicolumn{2}{|l|}{ UV protection } \\
\hline Sunscreen & $30(100)$ \\
\hline Hat & $0(100)$ \\
\hline Eyeglasses & $0(100)$ \\
\hline Umbrella & $4(13.3)$ \\
\hline \multicolumn{2}{|l|}{ Outdoor activity } \\
\hline Going to work & $30(100)$ \\
\hline Early morning sunbathing & $10(33.3)$ \\
\hline \multicolumn{2}{|l|}{ Using tretinoin cream $0.025 \%$} \\
\hline Routine & $30(100)$ \\
\hline Not routine & $0(0)$ \\
\hline
\end{tabular}

$\mathrm{UV}=$ Ultraviolet

Table 2. Results of Janus evaluation results on wrinkles

\begin{tabular}{lrr}
\hline & $\begin{array}{r}\text { Range } \\
\end{array}$ & Mean \pm SD \\
\hline Janus 3 $(\mathrm{n}=30)$ & $1-18$ & $7.03 \pm 3.65$ \\
Janus 4 $(\mathrm{n}=30)$ & $2-27$ & $9.5 \pm 5.93$ \\
Janus 5 $(\mathrm{n}=30)$ & $2-29$ & $8.23 \pm 4.87$ \\
\hline
\end{tabular}

Friedman test; $p=0.212$

Table 3. Results of Janus evaluation results on black spots

Range $\quad$ Mean \pm SD

(Minimum-Maximum)

\begin{tabular}{lrr}
\hline Janus 3 $(\mathrm{n}=30)$ & $12-47$ & $31.13 \pm 8.74$ \\
Janus 4 $(\mathrm{n}=30)$ & $17-48$ & $32.63 \pm 8.17$ \\
Janus 5 $(\mathrm{n}=30)$ & $18-47$ & $31.43 \pm 7.66$ \\
\hline
\end{tabular}

Anova test; $\mathrm{p}=0.694$ 
Table 4. Results of Janus evaluation results on UV black spots

Range

Mean \pm SD

(Minimum-Maximum)

\begin{tabular}{lrr}
\hline Janus $3(\mathrm{n}=30)$ & $2-28$ & $9.27 \pm 6.37$ \\
Janus $4(\mathrm{n}=30)$ & $1-27$ & $8.57 \pm 6.56$ \\
Janus 5 $(\mathrm{n}=30)$ & $2-24$ & $8.9 \pm 5.89$ \\
\hline
\end{tabular}

Anova Test; $p=0.317$

Table 5. Results of Janus evaluation results on skin tone

\begin{tabular}{lrr}
\hline & $\begin{array}{r}\text { Range } \\
\end{array}$ & Mean \pm SD \\
\hline Janus 3 $(\mathrm{n}=30)$ & $27-38$ & $33 \pm 2.77$ \\
Janus 4 $(\mathrm{n}=30)$ & $27-38$ & $32.6 \pm 2.66$ \\
Janus 5 $(\mathrm{n}=30)$ & $26-38$ & $32.53 \pm 2.75$ \\
\hline
\end{tabular}

Anova Test; $p=0.065$

Table 6. Results of Janus evaluation results on pores

\begin{tabular}{lrr}
\hline & $\begin{array}{r}\text { Range } \\
\end{array}$ & Mean \pm SD \\
\hline Janus 3 $(\mathrm{n}=30)$ & $38-59$ & $49.37 \pm 5.05$ \\
Janus 4 $(\mathrm{n}=30)$ & $31-60$ & $49.46 \pm 5.44$ \\
Janus 5 $(\mathrm{n}=30)$ & $38-57$ & $49.83 \pm 4.85$ \\
\hline
\end{tabular}

Friedman Test; $\mathrm{p}=1$

Table 7. Side effects and adverse effects after participating in the study

\begin{tabular}{ll}
\hline Side effects and adverse effects & $\mathrm{n}(\%)$ \\
\hline Persistent erythema & $0(0)$ \\
Itchy & $0(0)$ \\
Post-inflammatory hyperpigmentation & $0(0)$ \\
Hypopigmentation & $0(0)$ \\
Scarring & $0(0)$ \\
Infection & $0(0)$ \\
None & $30(100)$ \\
\hline
\end{tabular}

\section{DISCUSSION}

This study was an observational cohort conducted in 30 study samples. This study aimed to evaluate the long-term effects of topical mixture of AMSC-MP with vitamin $\mathrm{E}$ and $\mathrm{CO}_{2}$ fractional laser as LADD on photoaged skin. All research subjects were given topical PM-AMSC mixture with vitamin E after $\mathrm{CO}_{2}$ fractional laser, three times with an interval of 1 to 2 months from January to April 2019. Then an evaluation was carried out every month for three months. Long-term evaluation is obtained through the results of Janus skin analyzer for wrinkles, polarized black spots, UV black spots, skin tone, and pores.

In an Australian study of subjects before 30 years of age, moderate to severe photoaging was found in $72 \%$ of men and $47 \%$ in women. ${ }^{11}$ All participants were purposively females to uniform the data and reduce the drop out rate. Furthermore, in general, women concerned about skin care and rejuvenation more than men. This was supported by patients' data of the Cosmetic Division, Dermatology and Venereology Outpatient Clinic Dr. Soetomo Hospital, which in 2018, there were 711 female patients and 24 male patients of skin rejuvenation treatment.

The participants were grouped according to age range in accordance with the 2009 Indonesia Health Ministry criteria. There were 11 participants (36.7\%) in $46-55$ age group, 10 participants $(33.3 \%)$ in $36-45$ age group, and 9 participants $(30 \%)$ in 56-65 age group. The youngest participant was 38 years old, and the oldest was 62 years old. This was slightly different than the result of the study conducted by Malvy et al. in 12,735 participants aged 45-60 years old in France. A study found that the age group of 55-60 years old had the highest prevalence of photoaging. ${ }^{3}$ Photoaging has onset as early as late teens, whereas intrinsic aging generally begins at 50 years of age. Photoaging 
is directly related to cumulative sun exposure and aging. ${ }^{11,12}$

These participants of the study had either Glogau type 2 or 3 . There were $16(53,3 \%)$ participants with Glogau type 3 and $14(46,7 \%)$ participants with Glogau type 2. Gloglau type 2 is moderate photoaging occurring in the late $30 \mathrm{~s}$ to $40 \mathrm{~s}$. Glogau type 2 characters include wrinkles due to facial expression movements. Glogau type 3, which is advanced photoaging, generally occurs at the age of 50 characterized by resting wrinkles. Patients with Glogau type 2 and 3 photoaging are more likely to complain due to facial photoaging problems. Patients with Glogau type 1 do not consider that photoaging therapy is necessary. It is difficult to repair photoaged skins with Glogau type 4, severe photoaging.

Fitzpatrick's skin type evaluation revealed that there were $16(53.3 \%)$ participants with type 5 skins and $14(46.7 \%)$ participants with type 4 skins. Photoaging was more common in patients with brighter skin. Patients with Fitzpatrick type 1, 2, and 3 are more likely to suffer from photoaging than type 4, 5 , and 6 due to low melanocyte count as sun protection. In addition, geographic factors such as higher altitude and equatorial proximity also contribute to photoaging where the harmful effects of ultraviolet are higher. Habitual prolonged sun exposure due to outdoor activities also contributes to photoaging. Occupations with extensive sun exposure such as farmers, sailors, and truck drivers and indoor tanning promote photoaging as well. ${ }^{11}$

Tretinoin is the main therapy for patients with mild to moderate photoaging. Tretinoin use should be started at low concentrations, then slowly increased to avoid irritations. Griffiths compared $0.1 \%$ with $0.025 \%$ tretinoin use for eight months and found that differences in epidermal changes were not significant. ${ }^{13}$ All research participants received routine tretinoin $0.025 \%$ as maintenance therapy. This was to prevent skin irritations and minimize research bias.

Janus 3, 4, and 5 evaluations revealed that there was no significant difference in wrinkles. This suggested that the therapy had long-term effects. Lee et al. who compared micro-needling and microneedling with therapeutic products of stem cell metabolites found significant differences on wrinkle repair. ${ }^{14}$ Vitamin $\mathrm{E}$ reduces ROS generation, and reduces wrinkle formation. ${ }^{15}$

Long-term effects on wrinkles in this result may be caused by the combination therapy of AMSC-MP and vitamin E. Product metabolites of stem cell can improve wrinkles due to a wide variety of growth factors that can stimulate the synthesis of collagen, stimulate the proliferation and migration of dermis fibroblasts, epidermal keratinocytes, and increase fibroblast synthesis.

Janus 3, 4, and 5 evaluations on polarized black spots and UV black spots in this study found no significant changes. This suggested that the therapy had long-term effects. Study by Zhou et al. on a conditioned medium from adipose-derive stem cell (ADSC-CM) therapy using fractional $\mathrm{CO}_{2}$ laser found decreased hyperpigmentation after the therapy. ${ }^{16}$ Lee et al. also found significant improvement in the melanin index after micro-needling therapy with stem cell metabolite products. ${ }^{14}$ Vitamin $\mathrm{E}$ also has an active photoprotection effect that may play a role in repairing black spots due to photoaging. ${ }^{4}$

The treatment of a combination of AMSC-MP and vitamin $\mathrm{E}$ revealed long-term effects on polarized black spots and UV black spots. Stem cell metabolite products have antioxidant activities on the whitening growth factor. This can inhibit melanin synthesis, tyrosinase activity, and decrease the expression of melanogenic enzymes. The photoprotective effect of vitamin E may also play a role in the long-term effects in polarized and UV black spots.

Janus 3, 4, and 5 evaluations on skin tones showed no significant changes, which indicates that the therapy had long-term effects on skin tone. The treatment of a combination of AMSC-MP and vitamin E revealed long-term effects on skin tones. Stem cell metabolite products have whitening properties that work by inhibiting melanin formation, changing the shape of melanin, and affecting the distribution of melanosome transfer. TGF- $\beta 1$ can inhibit pigment formation by influencing the synthesis of tyrosine. Photoprotective effects of vitamin E can also affect the skin tone.

Janus 3, 4, and 5 evaluations found no significant changes in pores. This suggested that the therapy had long-term effects on pores. The definition of pores is unclear and tends to be interpreted as the opening of the pilosebaceous unit. Pores are normal structures but can be a cosmetic problem if there is an increase in size. An increase in pore size is assumed due to the effect of aging. In addition, index elasticity is also a factor that relates to wrinkles due to aging. Increased pores are said to be associated with increased wrinkles. ${ }^{17}$

Both stem cell metabolic products and vitamin $\mathrm{E}$ can promote pore repair. AMSC-MP contains growth factors that can stimulate collagen synthesis, stimulate proliferation and migration of dermal fibroblasts, epidermal keratinocytes, and enhance fibroblast synthesis. This can improve skin elasticity, which then promotes pores repair. Antioxidant properties of vitamin $\mathrm{E}$ counteract ROS, which then prevents 
collagen degradation, improving skin elasticity, and then repair the pores.

There was no side or adverse effect observed during the three months of evaluation. AMSC-MP is a fairly safe therapy because it is acellular, so it's easier to control than products that contain cells when applied to wounds. ${ }^{18}$ Vitamin E can function as an anti-inflammatory agent and influence the remodeling of scars by interacting with phospholipids on cell membranes, inducing molecular, and maintaining membrane stability. Vitamin $\mathrm{E}$ is used to improve wound healing, prevent hypertrophic scars, and reduce itching. To date, there has been no consensus or proper evidence of vitamin $\mathrm{E}$ use in treating wounds. ${ }^{19}$

The low energy of $\mathrm{CO}_{2}$ fractional laser and tretinoin use in low on centration can minimize the possibility of side effects and adverse effects due to fractional laser. Previous research on the effects of conditioned media from adipose-derived stem cells (ADSC-CM) on wound healing after $\mathrm{CO}_{2}$ fractional laser conducted by Zhou et al. confirmed the good results. A study concluded that the ADSC-CM application could effectively improve wound healing after fractional $\mathrm{CO}_{2}$ laser by reducing the temporary side effects of the laser such as erythema, hyperpigmentation, and increased TEWL. ${ }^{16}$

The limitation of this study was the uncertainty of whether the long-term effects were not influenced by other factors such as maintenance therapy or photoaging triggers or not. This study concluded that a combination of AMSC-MP and vitamin E delivered deeply using $\mathrm{CO}_{2}$ fractional laser provided long-term effects on wrinkles, polarized black spots, UV black spots, skin tones, and pores due to photoaging, and there was no side and the adverse effect observed.

\section{REFERENCES}

1. Yin R, Chen Q, Hamblin MR. Skin Photoaging. San Rafael (CA): Morgan \& Caypool. 2014.

2. Gilchrest BA. Photoaging. J Invest Dermatol 2013; 133(E1): E2-6.

3. Malvy JM, Guinot C, Preziozi P, Vaillant L, et al. Epidemiology of determinant of skin photoaging: baseline data of the SU.VI.MAX. cohort. J Am Acad Dermatol 2000; 42(1): 47-55.

4. Thiele JJ, Ekanayake-mudiyanselage S. Vitamin $\mathrm{E}$ in human skin: organ-specific physiology and considerations for its use in dermatology. Mol Aspects Med 2007; 28(5): 646-67.

5. Gallardo V, Munoz M, Ruiz MA. Formulation of hydrogels and lipogels with vitamin E. J Cosmet Dermatol 2005; 4(3): 187-92.
6. Prakoeswa CRS, Pratiwi FD, Herwanto N, Citrashanty I, et al. The effects of Amniotic Membrane Stem Cell-Conditioned Medium on photoaging. J Dermatolog Treatment 2019; 30(5): 487-2.

7. Waibel JS, Rudnick A, Shagalov D, Nicolazzo DM. Update of ablative factional lasers to enhance cutaneous topical drug delivery. Adv Ther 2017; 34(8): 1840-9.

8. Zalerski-Larsen LA, Fabi SG. Laser-assisted drug delivery. Dermatol Surg 2016; 42(8): 91931 .

9. Issa MCA, Torreao PS. Transdermal drug delivery with ablative methods. In: Issa MCA, Tamura B, editors. Lasers, lights and other technologies. Cham: Springer. 2018. P. 463-9.

10. Ibrahim O, Wenande E, Hogan S, Arndt KA, et al. Challenges to laser-assisted drug delivery: applying theory to clinical practice. Lasers Surg Med 2017; 50(1): 20-7.

11. Han A, Chien AL, Kang S. Photoaging. Dermatol Clin 2014; 32(3): 291-9.

12. Farage M A, Miller KW, Elsner P, Maibach HI. Intrinsic and extrinsic factors in skin aging: a review. Int J Cosmet Sci 2008; 30(2): 87-95.

13. Hubbard BA, Unger JG, Rohrich RJ. Reversal of skin aging with topical retinoids. Plast Reconstr Surg 2014; 133(4): 481-90.

14. Lee HJ, Lee EG, Kang S, Sung JH, Chung HM, Kim DH. Efficacy of microneedling plus conditioned medium of human stem cells for skin rejuvenation: a randomized, controlled, blinded, split-face study. Ann Dermatol 2014; 26(5): 584-91.

15. Telang PS. Vitamin $\mathrm{C}$ in dermatology. Indian Dermatol Online J 2013; 4(2): 143-6.

16. Zhou BR, Xu Y, Guo SL, Xu Y, Wang Y, Zhu $F$, et al. The effect of conditioned media of adipose-derived stem cells on wound healing after ablative fractional carbon dioxide laser resurfacing. Biomed Res Int 2013; 2-9.

17. Jung HJ, Ahn JY, Lee JI, Bae JY, Kim HL, Suh HY, et al. Analysis of the number of enlarged pores according to sit, age, and sex. Skin Res Technol 2008; 24(3): 367-70.

18. Tamama K, Kerpedjieva SS. Acceleration of wound healing by multiple growth factors and cytokines secreted from multipotential stromal cells/mesenchymal stem cells. Adv Wound Care 2012; 1(4): 177-82.

19. Tanaydin V, Conings J, Malyar M, van der Hulst $\mathrm{R}$, van der Lei $\mathrm{B}$. The role of topical vitamin $\mathrm{E}$ in scar management: a systematic review. Aesthet Surg J 2016; 36(8): 959-65. 